\title{
Über die Löslichkeit des schwefelsauren Ceroxyduls in Wasser.
}

\author{
Von \\ W. Muthmann und H. Rölig.
}

Mit 1 Figur im Text.

Bei der Trennung der Ceritmetalle, also von Cer, Lanthan, Neodym und Praseodym, spielt bekanntlich die Löslichkeit gewisser Salze eine grofse Rolle. So wird z. B. nach Bunsen's ${ }^{1}$ Vorschlag das Lanthan von den übrigen Erden in der Weise getrennt, dals die wasserfreien Sulfate in Eiswasser eingetragen werden und die Lösung langsam auf $30^{\circ}$ erwärmt wird, es soll dann reines wasserhaltiges Lanthansulfat sich abscheiden, während die übrigen Metalle in Lösung bleiben.

Das Cer wird am besten gereinigt durch wiederholtes Umkrystallisieren des Ceriammoniumnitrats, und die Spaltung des Didyms in Neodym und Praseodym gelang bekanntlich nur durch fortgesetztes Umkrystailisieren der Ammoniumdoppelnitrate.

In den angeführten Fällen beruht die Trennung also auf der verschiedenen Löslichkeit der betreffenden Salze, und es scheint, dals die Methode des Umkrystallisierens bei den seltenen Erden im allgemeinen am schnellsten zu einer Trennung führt. Um sicher zu gehen und die Methode beurteilen zu können, ist unseres Erachtens nach ein eingehendes Studium und möglichst vollkommene Kenntnis der Löslichkeitserscheinungen bei den betreffenden Salzen unbedingtes Erfordernis. Man sollte, bevor man eine solche Trennungsmethode versucht, zunächst die Löslichkeit des Salzgemisches und wenn irgend möglich, auch diejenige der Einzelsalze kennen; je gröfser die Löslichkeitsdifferenzen sind, um so schneller wird man zum Ziele gelangen, und eine mittlere Löslichkeit erleichtert das Umkrystallisieren ungemein.

1 Pogg. Ann. 155, 377. 
Leider ist es nun mit unseren Kenntnissen in dieser Beziehung noch recht schlecht bestellt und glaubten wir daher eine Lücke in der Chemie der Ceriterden auszufüllen, wenn wir von einigen wichtigen Salzen Löslichkeitsbestimmungen ausführten. Eine Anzahl derartiger Untersuchungen sind im Gange, in dieser Arbeit möchten wir nur das Cerosulfat besprechen, das merkwürdige und theoretisch wichtige Erscheinungen zeigt.

\section{Darstellung des Materials.}

Das von uns untersuchte Salz war dargestellt aus schwedischem Cerit nach der bekannten Methode. In Arbeit genommen wurden $15 \mathrm{~kg}$ Cerit; derselbe wurde geglüht, mit konzentrierter Salzsäure aufgeschlossen, die Oxalate aus saurer Lösung gefällt, verglüht und das Erdengemisch in Salpetersäure aufgelöst. Dann wurde Ammonnitrat in geringem Überschusse zugesetzt, bis zur völligen Lösung erhitzt, und das in prachtvollen, orangeroten Krystallen ausgefallene Ceriammonnitrat zehnmal in der Weise umkrystallisiert, dafs das mit einem Pukar'schen Thonfilter trocken gesaugte Salz in wenig heifsem Wasser gelöst und dann mit konzentrierter Salpetersäure gefällt wurde.

Nach früheren Untersuchungen, namentlich von B. BRAUner, durften wir annehmen, dals das so erhaltene Produkt frei von Lanthan und Didym war. Das Lanthan bildet ja keine Verbindung, die in ihrer Zusammensetzung dem Ceriammonnitrat entspricht. Eine Methode, kleine Lanthanmengen nachzuweisen, existiert ja leider nicht, die Methode der Spektralanalyse versagt, wenn der Lanthangehalt unter einen gewissen Prozentsatz sinkt. Das Spektrum unserer Verbindung, nach LECOCQ DE BOIBEAUDRAN mit einem Apparat mit drei Prismen untersucht, zeigte keine Lanthanlinien.

Eine Titration des Cers in dem sehr fein krystallinisch abgeschiedenen, unter dem Mikroskop ganz einheitlich aussehenden Salze ergab die Formel $\mathrm{Ce}\left(\mathrm{NO}_{3}\right)_{4} \cdot 2 \mathrm{NH}_{4} \mathrm{NO}_{3}+\mathrm{H}_{2} \mathrm{O}$. Das Salz wurde in Jodkaliumlösung eingetragen, und das ausgeschiedene Jod schnell mit 'Thiosulfatlösung titriert. $0.4723 \mathrm{~g}$ verbrauchten $8.05 \mathrm{~cm}$ einer Lösung vom Sauerstofftiter 0.000839 ; mithin gefunden $30.42 \%$ $\mathrm{CeO}_{2}$; für obige Formel berechnet $30.41 \%$. Holzmann fand $1 \frac{1}{2}$ Mol. $\mathrm{H}_{2} \mathrm{O}$ (ber. $29.92 \% \quad \mathrm{CeO}_{2}$ ); wahrscheinlich war der von ihm untersuchte Körper nicht ganz frei von anhängender Feuchtigkeit. Von einer direkten Wasserbestimmung, die wegen des Ammoniaks sehr schwierig ist, wurde abgesehen. 
Nach dem Reduzieren der Ceriverbindung durch Kochen mit etwas Alkohol resultierte eine völlig farblose Flüssigkeit, die auch in $8 \mathrm{ccm}$ dicker Schicht keine Absorptionslinien zeigte. Diese Lösung wurde mit Oxalsäure gefällt, und das Oxalat in einem Nickeltiegel verglüht; das erhaltene Dioxyd war völlig weils, auch ein Beweis für die Abwesenheit von Didymerden, die bekanntlich, selbst wenn sie nur in Spuren zugegen sind, dem Oxyde eine fleischrote bis ziegelrote Farbe erteilen. Wir dürfen also behaupten, dafs unser Cerpräparat allen Anforderungen entspricht, die man an ein reines Präparat stellen kann. Das so erhaltene Cerdioxyd ist ein aufserordentlich beständiger Körper. $\mathrm{Er}$ ist, wie gesagt, bei gewöhnlicher Temperatur rein weils, wird beim Erhitzen citronengelb, beim Erkalten wieder weifs. Er wird im Wasserstoffstrom auch bei beginnender Gelbglut nicht reduziert, alle Angaben in der Litteratur sind falsch, die besagen, es könne $\mathrm{Ce}_{2} \mathrm{O}_{3}$ dargestellt werden durch Glühen von $\mathrm{CeO}_{2}$ im Wasserstoffstrom. Es wird nicht verändert beim Kochen mit Salpetersäure, Salzsäure oder verdünnter Schwefelsäure, auch nicht nach Zusatz von schwefliger Säure oder Alkohol; angesäuerte Jodkaliumlösung jedoch reduziert langsam beim Kochen unter Jodabscheidung.

Die Überführung in Cerosulfat geschieht am besten durch $\mathrm{Ab}$ rauchen mit konzentrierter Schwefelsäure und Erhitzen des orangeroten, in der Hitze braunroten Cerisulfats auf $600^{\circ}$. Es bleibt rein weifses $\mathrm{Ce}_{2}\left(\mathrm{SO}_{4}\right)_{3}$, das beim Eintragen in Eiswasser unter Schütteln sich völlig klar auflöst. Dieses Produkt wurde bei den $z u$ beschreibenden Löslichkeitsbestimmungen verwendet.

\section{Die aus rein wässeriger Lösung entstehenden Hydrate des Cerosulfats.}

In der Litteratur ${ }^{1}$ finden sich Angaben über nicht weniger als fünf verschiedene Hydrate des Cerosulfats; das Salz soll mit 5, 6, 8,9 und $12 \mathrm{Mol}$. $\mathrm{H}_{2} \mathrm{O}$ krystallisieren können. Von diesen konnten wir indessen nur drei erhalten; bei unseren zahlreichen Versuchen wurden niemals Salze mit 6 und 9 Mol. Wasser beobachtet.

Die Nichtexistenz dieser beiden Hydrate können wir natürlich nicht mit voller Sicherheit behaupten, es ist möglich, dals sie aus schwefelsaurer Lösung von bestimmtem Säuregehalt entstehen. Jedenfalls orhält man sie aus Wasser bei Anwendung reinen Cero-

1 Dammer's Handbuch 3, 20. 
sulfats nicht; wir vermuten aus Gründen, die in einer späteren $A b$ handlung dargelegt werden sollen, dafs dieses Salz nur dann mit 6 oder 9 Mol. $\mathrm{H}_{2} \mathrm{O}$ krystallisiert, wenn es noch Lanthan beigemengt enthält. Zunächst möchten wir die drei sicher beobachteten Hydrate kurz beschreiben.

1. $\mathrm{Ce}_{2}\left(\mathrm{SO}_{4}\right)_{3} \cdot 5 \mathrm{H}_{2} \mathrm{O}$. Dieser Körper bildet schöne, luftbeständige, prismatische Kryställchen, welche regelmälsig zu Büscheln vereinigt sind. Das System ist wahrscheinlich das monokline; sämtliche von uns beobachtete Krystalle waren Zwillinge; unter dem Mikroskop zwischen gekreuzten Nicols erscheint nämlich jedes Prisma der Länge nach in zwei Hälften geteilt, von denen jede verschieden auslöscht und zwar in der Weise, dafs die Auslöschungsrichtungen symmetrisch schief zur Längsaxe stehen. Oft kommt es auch vor, dafs die Zwillingsgrenze unregelmäfsig im Krystalle verläuft, ähnlich wie bei den wohlbekannten Quarzzwillingen. Die Analyse wurde in diesem, wie in allen folgenden Fällen in der Weise ausgeführt, dals das Salz im Schwefelbade, also bei $440^{\circ}$, erhitzt und der Wasserverlust bestimmt wurde. Schwefelsäure geht bei dieser Temperatur nach BRAUNER' ${ }^{1}$ eingehenden Untersuchungen bekanntlich nicht verloren.

$$
\begin{gathered}
\text { Analyse: } \\
0.4790 \mathrm{~g} \text { Substanz verloren } 0.0645 \mathrm{~g} \mathrm{H}_{2} \mathrm{O}=13.46 \% \\
0.5830 \mathrm{~g} " \text { B } " \text { " } 0.0790 \mathrm{~g} \mathrm{H}_{2} \mathrm{O}=13.55 \% \\
\text { Berechnet für } 5 \mathrm{H}_{2} \mathrm{O}=13.67 \% \mathrm{H}_{2} \mathrm{O} .
\end{gathered}
$$

Das Pentahydrat entsteht immer in neutraler Lösung, wenn die Temperatur höher als $74^{\circ}$ ist; wir erhielten es einfach durch Eindampfen verdünnter Lösungen auf dem Wasserbade: Andere Hydrate sind dem Salze, wenn es so dargestellt ist, nicht beigemengt.

2. $\mathrm{Ce}_{2}\left(\mathrm{SO}_{4}\right)_{3} 8 \mathrm{H}_{2} \mathrm{O}$. Die schönen rhombischen Pyramiden, welche entstehen, wenn man Cerosulfatlösungen zwischen 30 und $74^{\circ}$ eindunsten läfst, bilden dieses Hydrat. Das Sulfat des Handels, gewöhnlich durch Didymsalze rötlich gefärbt, enthält ebenfalls 8 Mol. Wasser. Dasselbe ist des öfteren analysiert und von MARIGNAC $^{2}$ krystallographisch bestimmt worden. Wir erhielten leicht Krystalle bis zu $1 \mathrm{~cm}$ im Durchmesser. Unter dem Mikroskope ist es charakterisiert durch seine Eigenschaft, immer in einzelnen

1 Monatsh. Chem. 6, 793.

2 Recherch. sur les fermes cristalles de quelques composés chemiques (Genéve 1855) S. 37.

Z. anorg. Chem. XVI. 
Pyramiden zu krystallisieren, während das vorhergehende büschelförmige Aggregate bildet; die beiden Körper sind nicht zu verwechseln.

$$
\text { Analyse: }
$$

$0.2835 \mathrm{~g}$ verloren $0.0555 \mathrm{~g} \mathrm{H}_{2} \mathrm{O}=19.57 \%$

$0.4545 \mathrm{~g} \quad, \quad 0.0900 \mathrm{~g} \mathrm{H}_{2} \mathrm{O}=19.80$ "

Berechnet für $\mathrm{Ce}_{2}\left(\mathrm{SO}_{4}\right)_{3} .8 \mathrm{H}_{2} \mathrm{O}=20.23 \%$

Die Krystalle waren manchmal etwas trübe, einige ganz klare wurden zur Schwefelsäure- und Cer-Bestimmung benutzt.

$\begin{array}{lcc} & \text { Gefunden: } & \text { Berechnet für } \\ \mathrm{Ce}_{2} \mathrm{O}_{2} \mathrm{O} \text { : } & 45.90 & 46.07 \\ \mathrm{SO}_{3} & 34.01 & 33.71\end{array}$

Man findet leicht etwas Wasser zu wenig, wohl infolge des Trübewerdens der Krystalle an der Luft.

3. $\mathrm{Ce}_{2}\left(\mathrm{SO}_{4}\right)_{3}+12 \mathrm{H}_{2} \mathrm{O}$. Wenn man reine Cerosulfatlösungen bei gewöhnlicher Temperatur verdunsten läfst, so scheiden sich zuerst meistens die Pyramiden mit $8 \mathrm{H}_{2} \mathrm{O}$ aus, oft sieht man aber neben diesen noch merkwürdige Bildungen, welche ganz genau so aussehen, wie Schimmelpilzkolonien und von uns auch zuerst dafür gehalten wurden; diese aus Krystallfäden bestehenden Formen sind das Dodekahydrat. Nur von Jourv ist dasselbe bis jetzt beobachtet worden; er beschreibt es als ,asbestartige Nadeln", doch trifft das nieht ganz zu, denn die einzelnen Kryställchen sind noch feiner als Asbestkrystalle, sie erreichen meist noch nicht $1 / 25$ des Durchmessers von einem Menschenhaar. Sehr bequem erhält man diese Form in folgender Weise: Wasserfreies Cerosulfat wird unter fleilsigem Schütteln in Eiswasser eingetragen. Es findet dann Auflösung statt und zwar können 100 Teile Wasser 40, ja nach Brauner's ${ }^{1}$ Beobachtungen bis zu 60 Teile $\mathrm{Ce}_{2}\left(\mathrm{SO}_{4}\right)_{3}$ lösen. Hat man 20 Gewichtsteile Salz auf 100 Teile Wasser gelöst und fährt mit dem Eintragen und Schütteln fort, so bemerkt man über kurz oder lang, wie die Flüssigkeit plötzlich zu opalisieren anfängt; es ist dies das Zeichen für den Beginn der Ausscheidung des Dodekahydrats. Man stellt dann das Gefäls in Eis und wartet etwa 15 Minuten; die Flüssigkeit ist dann völlig angefüllt mit den beschriebenen feinen Haaren und erstarrt dann zu einem krystallinischen Magma. Der Körper wurde sorgfältig abgeprefst und analysiert.

1 Journ. chem. Soc. 53, 357. 


$$
\begin{gathered}
\text { Analyse: } \\
0.3445 \mathrm{~g} \text { Substanz verloren } 0.0955 \mathrm{~g} \mathrm{H}_{2} \mathrm{O}=27.72 \% \\
\text { Berechnet für } \mathrm{Ce}_{2}\left(\mathrm{SO}_{4}\right)_{3} .12 \mathrm{H}_{2} \mathrm{O}=27.55 \%
\end{gathered}
$$

Joun's Angabe ist also richtig.

\section{Löslichkeitsbestimmungen.}

Man kann bei Untersuchungen, wie die vorliegende, nur dann auf verständliche Resultate rechnen, wenn man zwei wichtige Sätze sich vergegenwärtigt; nämlich:

1. eine Lösung kann gesättigt sein nur in Bezug auf einen festen Körper, mit dem sie in Berührung ist, und

2. bei einem System: 2 Stoffe $\left(\mathrm{H}_{2} \mathrm{O}\right.$ und Salz) besteht Gleichgewicht bei Anwesenheit von 3 Phasen (Bodenkörper, Lösung, Dampf). Zwei Bodenkörper koëxistieren bei einer ganz bestimmten Temperatur, und diese Temperatur ist die Umwandlungstemperatur.

Leider scheinen diese wichtigen Folgerungen aus GIBBs' Phasenregel bei einigen Chemikern noch nicht völlig anerkannt zu werden (siehe die folgende Abhandlung).

Es ist also im vorliegenden Falle, wo drei Hydrate existieren, durchaus sinnlos, von Löslichkeit des Cerosulfats schlechthin zu sprechen; neben der Temperatur muls man noch angeben, welches Hydrat als Bodenkörper vorhanden war. Geschieht dieses nicht, so ist die Untersuchung einfach wertlos.

Bei der praktischen Ausführung ist es vor allem wichtig, genau darauf zu achten, dafs am Boden der zu analysierenden Lösung nicht zwei verschiedene Hydrate sich befinden. Man hätte dann (wenn nicht zufällig die Temperatur mit dem Umwandlungspunkte zusammentrifft) kein im Gleichgewicht befindliches System; beim Stehenlassen mülste sich das eine Hydrat in das andere, bei der betreffenden Temperatur stabile, umwandeln, und so lange dieser Prozels davert, würde sich die Konzentration im einen oder anderen Sinne ändern. Die Ausdrücke „Löslichkeit" und ,gesättigte Lösung“ können sich nur beziehen auf Systeme, welche, Konstanz von Temperatur und Druck vorausgesetzt, sich unverändert halten. Selbstredend kann das Gleichgewicht auch ein labiles sein, und wir werden sogleich Beispiele von solch labilen Gleichgewichten kennen lernen. Wir lassen die Resultate unserer Analysen folgen:

1. Das Pentahydrat. Die Bestimmungen wurden folgendermalsen durchgeführt: In einem Thermostaten von $15 \mathrm{l}$ Inhalt, in dem die Temperatur leicht stundenlang auf $1^{\circ}$ konstant gehalten 
werden konnte, wurde ein Becherglas mit destilliertem Wasser eingesenkt, und dann unter fortwährendem Rühren mit der bekannten WiTr'schen Vorrichtung das wie oben dargestellt reine Pentahydrat eingetragen. Löste sich nichts mehr auf, so liels man absitzen, analysierte ca. $1 \mathrm{ccm}$ der Flüssigkeit (Eindunsten und Entwässern im Schwefelbade bis zur Gewichtskonstanz), setzte das Rühren noch $1 / 2$ Stunde fort und untersuchte eine zweite Probe. Regelmälsig gaben beide Proben fast genau dieselben Werte. Im ganzen wurden fünf Bestimmungen ausgeführt.

\begin{tabular}{c|c|c}
\hline $\begin{array}{c}\text { Nr. } \\
\text { des } \\
\text { Versuches }\end{array}$ & $t$ & $\begin{array}{c}\text { Gew.-Teile } \mathrm{Ce}_{2}\left(\mathrm{SO}_{4}\right)_{3} \\
\text { auf 100 Teile } \mathrm{H}_{2} \mathrm{O}\end{array}$ \\
\hline \hline & $100^{\circ}$ & 0.775 \\
2 & $80^{\circ}$ & 1.70 \\
3 & $60^{\circ}$ & 3.45 \\
4 & $50^{\circ}$ & 5.56 \\
5 & $40^{\circ}$ & 8.20
\end{tabular}

Die Löslichkeit nimmt also mit abnehmender Temperatur stark $\mathrm{zu}$; sie ist bei $40^{\circ}$ zehnmal so grols, als bei $100^{\circ}$. Über $40^{\circ}$ hinaus konnten keine Zahlen mehr erhalten werden; die Umwandlung in das Oktohydrat erfolgte nach ganz kurzem Stehen. Eine Bestimmung bei $30^{\circ}$ auszuführen, war uns nicht möglich.

2. Das Oktohydrat. Ausführung wie oben. Es wurden bestimmt sechs Punkte:

\begin{tabular}{c|c|c}
\hline $\begin{array}{c}\text { Nr. des } \\
\text { Versuches }\end{array}$ & $t$ & $\begin{array}{c}\text { Gew.Teile } \mathrm{Ce}_{2}\left(\mathrm{SO}_{4}\right)_{3} \\
\text { auf 100 Teile } \mathrm{H}_{2} \mathrm{O}\end{array}$ \\
\hline \hline & & \\
6 & $0^{\circ}$ & 19.10 \\
7 & $18^{\circ}$ & 17.32 \\
8 & $30^{\circ}$ & 16.13 \\
9 & $50^{\circ}$ & 12.48 \\
10 & $60^{\circ}$ & 9.40 \\
11 & $70^{\circ}$ & 4.24
\end{tabular}

Auch hier findet starke Abnahme der Löslichkeit mit steigender Temperatur statt. Bei $80^{\circ}$ findet so schnell die Umwandlung in 
das Pentahydrat statt, dafs keine Bestimmung mehr gemacht werden konnte.

3. Das Dodekahydrat. Man ging aus von der bei $0^{0}$ gesättigten Lösung, welche wie oben beschrieben erhalten worden war. Unter Schütteln wurde langsam die Temperatur erhöht und Proben der Flüssigkeit analysiert.

\begin{tabular}{c|c|c}
\hline $\begin{array}{c}\text { Nr. des } \\
\text { Versuches }\end{array}$ & $t$ & $\begin{array}{c}\text { Gew.-Teile } \mathrm{Ce}_{2}\left(\mathrm{SO}_{4}\right)_{3} \\
\text { auf } 100 \mathrm{Teile} \mathrm{H}_{2} \mathrm{O}\end{array}$ \\
\hline 12 & $0^{\circ}$ & 21.40 \\
13 & $18^{\circ}$ & 18.44 \\
14 & $25^{\circ}$ & 16.22
\end{tabular}

Oberhalb $25^{\circ}$ fand Umwandlung in das Oktohydrat statt.

\section{Diskussion der Resultate.}

Die beigedruckte Zeichnung giebt eine Übersicht über die erhaltenen Resultate; auf der Abscissenaxe sind, wie üblich, die Temperaturen, auf der Ordinatenaxe die Zusammensetzung der Lösungen eingetragen. $A B C$ ist die Löslichkeitskurve des Pentahydrats, $B D E$ die Okto- und $D F$ die des Dodekahydrats. Dafs die Löslichkeitsverhältnisse der drei Hydrate völlig verschieden sind, ersieht man aus der Zeichnung auf den ersten Blick, und könnte unser Beispiel als neuer Beweis für den oben angeführten Satz gelten, wenn ein solcher nicht nach den vielen schönen Versuchen von BAKHUIS-Roozeboom und von VAN'T HoFr gänzlich unnötig wäre.

Wie man sieht, wird die Kurve $B D E$ für das Oktohydrat zweimal geschnitten, bei $B$ und bei $D$. Bei ersterem Punkte ist die Temperatur $74^{\circ}$; die Löslichkeit ist dieselbe für das Okto- und Pentahydrat, die gesättigte Lösung enthält 2.4 Teile $\mathrm{Ce}_{2}\left(\mathrm{SO}_{4}\right)_{3}$ auf 100 Teile $\mathrm{H}_{2} \mathrm{O}$. Nur an diesem Punkte können beide Hydrate in Berührung mit der Lösung neben einander existieren; erhöht man die Temperatur, so findet Umwandlung in das Pentahydrat unter gleichzeitiger Ausscheidung von Substanz statt; erniedrigt man sie, so entsteht das Oktohydrat unter Auflösung, und man kommt in die Kurve $B E$ hinein. Diese letztere Umwandlung mufs stattfinden nur bei Gegenwart der rhombischen Krystalle des Hydrats mit $8 \mathrm{H}_{2} \mathrm{O}$; sind solche nicht zugegen, so erhält man die Fortsetzung von $A B$, nämlich $B C$. Dieser Kurventeil ist besonders interessant, 
er zeigt uns ein labiles Gleichgewicht des Systemes: $\mathrm{Ce}_{2}\left(\mathrm{SO}_{4}\right)_{3} \cdot 5 \mathrm{H}_{2} \mathrm{O}$, Lösung, Dampf. Labil ist auf den' sämtlichen Punkten der Linie das Gleichgewicht insofern, als das Eintragen der kleinsten Menge des Oktohydrats Umwandlung unter gleichzeitiger Auflösung her-

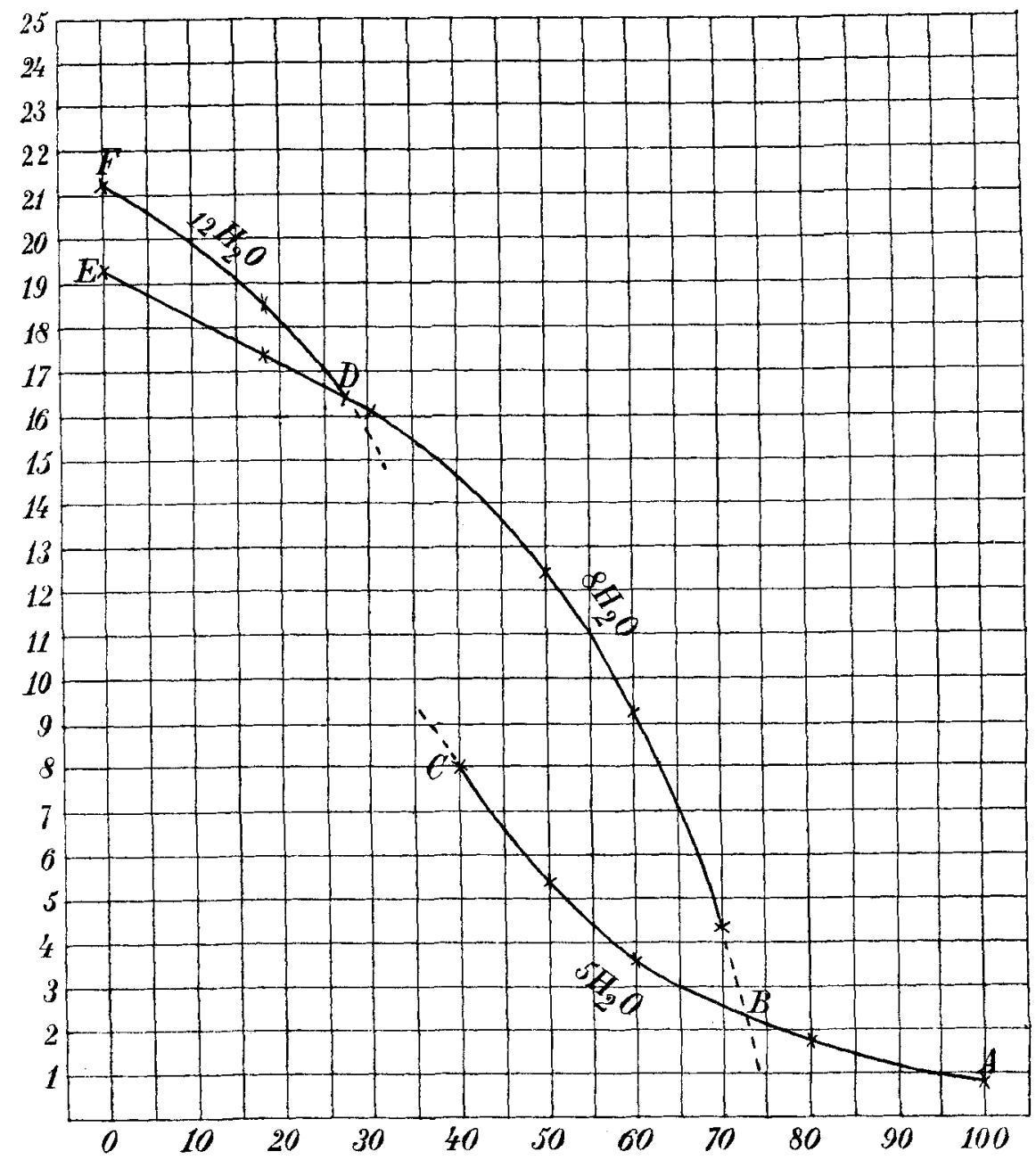

vorrult. Bei $50^{\circ} \mathrm{kann}$ man, wie ersichtlich, zwei gesättigte Lösungen von total verschiedenem Gehalt erzielen; das Oktohydrat ist bei dieser Temperatur mehr als doppelt so "leicht löslich" als das Pentabydrat. Beide Punkte sind von uns verifiziert worden.

Die Umwandlung des Pentahydrats in das Oktohydrat und umgekehrt, welche bei $72^{\circ}$ vor sich geht, erfolgt nur sehr träge. 
Wir haben mehrfach versucht diesen Punkt mit Hilfe des Dilatometers von van't How festzustellen, jedoch ohne Erfolg. Die Verwandlung erfolgt so langsam, dafs selbst bei äufserst vorsichtigem Anwärmen ein Ausschlag am Instrumente nicht zu beobachten ist.

Dagegen gelingt es, auf andere Weise diesen Punkt festzustellen; eine ca. $1 \%$ ige Lösung wurde in zwei Teile geteilt, und der eine bei $71^{\circ}$, der andere bei $75^{\circ}$ unter Rühren eingedampft. Im ersteren Falle erschienen Pyramiden des Salzes mit $8 \mathrm{H}_{2} \mathrm{O}$, im letzteren die Prismen des Pentahydrats. Der beste Beweis für den Umwandlungspunkt ist der Schnittpunkt der beiden Löslichkeitskurven.

Das Existenzgebiet des Dodekahydrats ist bei weitem beschränkter, als das der beiden anderen; daher kommt es auch, dal's es lange übersehen und erst kürzlich von JouIN entdeckt wurde. Der Umwandlungspunkt ist hier bei $27.5^{\circ}$; die Konzentration der Lösung $16.2 \mathrm{Ce}_{2}\left(\mathrm{SO}_{4}\right)_{3}$ auf 100 Teile Wasser. Die Löslichkeitskurve für das Oktohydrat konnte bis $0^{0}$ verfolgt werden; der Kurventeil $D E$ entspricht wieder einem labilen Gleichgewichte. Die Umwandlung in das Dodekahydrat erfolgt aber ganz aufserordentlich langsam; wir haben Lösungen, welche das Salz mit $8 \mathrm{H}_{2} \mathrm{O}$ enthielten, mit dem Dodekahydrat versetzt und 14 Tage lang bei $10^{\circ}$ stehen lassen, ohne dafs völlige Umwandlung erfolgt wäre. Allerdings wächst in solchen Lösungen, und das ist wichtig, bei $10^{\circ}$ nur das Dodekahydrat. Die Flüssigkeit erfüllt sich allmählich mit den weifsen Krystallfäden, während die am Boden festsitzenden Pyramiden scheinbar weder wachsen, noch an Menge abnehmen. Die Ursache dieser Erscheinung liegt jedenfalls mit an der Oberflächenbeschaffenheit der Krystalle, die kompakten Pyramiden bieten der Flüssigkeit nur eine verhältnismälsig kleine Oberfläche dar.

Dagegen erfolgt die Umwandlung des Dodekahydrats ganz glatt und kann thermometrisch verfolgt werden. Es mufs in diesem Falle Wärme gebunden werden, wie beim Schmelzen des Eises. Den Versuch führt man am bequemsten folgendermalsen aus:

Ein mit einem gewöhnlichen Normalthermometer versehener BECKManN'scher Schmelzpunktapparat wird mit dem bei $0^{\circ}$ erhaltenen Magma der Dodekahydratkrystalle beschickt und in einen gleichfalls auf $0^{\circ}$ gebrachten Thermostaten versenkt. Dann erhöht man die Temperatur ganz langsam und gleichmärsig, indem man sowohl die Flüssigkeit im Thermostaten, als auch die Substanz im BEckм.anv'schen Apparat fortwährend umrührt. Das Thermometer stieg gleichmälsig bis $28.5^{\circ}$, ging dann zurück auf $27.5^{\circ}$, blieb eine 
Zeit lang bei dieser Temperatur stehen, um dann weiter in die Höhe zu gehen. Als die Temperatur auf $32^{\circ}$ gestiegen war, wurde das Salz, das sich nunmehr leicht zu Boden setzte, mikroskopisch untersucht; die ganze Menge war in die rhombischen Pyramiden umgewandelt.

Der Umwandlungspunkt liegt also bei $27.5^{\circ}$, während in der Figur die Kurven sich bei $27^{\circ}$ schneiden; eine vorzügliche Übereinstimmung, wenn man bedenkt, dals die beiden Zahlen nach völlig von einander verschiedenen Methoden erhalten wurden.

\section{Das Verhalten des wasserfreien Cerosulfats.}

Das Anhydrid $\mathrm{Ce}_{2}\left(\mathrm{SO}_{4}\right)_{3}$ zeigt bei der Auflösung eine ganz auffallende Ähnlichkeit mit dem wasserfreien Thoriumsulfat, das von Baknuis-RoozeBoom eingehend erforscht worden ist. Schon oben, bei der Beschreibung des Dodekahydrats, sind die Erscheinungen beschrieben worden; trägt man die Substanz in Eiswasser unter Schütteln ein, so scheint die Löslichkeit eine unbegrenzte, bis plötzlich das Opalisieren sich zeigt und Dodekahydrat gebildet wird.

Die Ursache dieser merkwürdigen und wichtigen Erscheinung ist jedenfalls dieselbe, die BAKнUтs-Roоzввоом für das $\mathrm{ThS}_{2} \mathrm{O}_{8}$ annimmt. Das System: gesättigte Lösung, Anhydrid, Dampf ist bei allen Temperaturen zwischen $0-100^{\circ}$ labil und zwar in Bezug auf Hydrate, die in den angegebenen Grenzen erhalten werden; sie ist ferner um so labiler, je höher die Temperatur ist, denn schon bei $20^{\circ}$ gelingt es nicht mehr von dem Anhydrid überhaupt beträchtlichere Mengen aufzulösen, ohne dafs Hydratisierung eintritt. Jedenfalls wird auch hier irgendwo oberhalb $100^{\circ}$ ein Punkt existieren, an dem sich das Pentahydrat oder ein anderes, noch unbekanntes mit weniger Wasser, in das Anhydrid verwandelt, und von diesem Punkte ab würde man dann stabile Systeme in Berührung mit dem Anhydrid erhalten. Doch sind von uns der experimentellen Schwierigkeiten halber - man hätte natürlich unter hohem Drucke zu arbeiten - Versuche in dieser Richtung nicht gemacht worden.

\section{Bemerkung zu der Abhandlung des Herrn W. W. J. NICOL: ${ }^{1}$ "Übersättigung und ihre Abhängigkeit von der Krystallform."}

Vor kurzem hat Herr Nrcor eine eigentümliche Ansicht ausgesprochen, auf die wir hier etwas einzugehen um so mehr Veran-

${ }^{1}$ Z. anorg. Cherr. 15, 397. 
lassung haben, als der eine von uns sich lange Zeit mit dem Phänomene der Krystallisation beschäftigt hat; es wird nämlich behauptet, dafs nur dann Übersättigung und Überschmelzung eintreten können, wenn, unter den Bedingungen des Experimentes, der gelöste Körper in mehreren allotropen Modifikationen auftreten kann.

Wir können uns mit dieser Behauptung ganz und gar nicht einverstanden erklären; schon das einfachste Beispiel des bestuntersuchten Körpers, nämlich des Wassers, lehrt dies. Niemals ist bei $-5^{0}$ aulser der hexagonalen eine zweite Modifikation des Eises beobachtet worden, und wie leicht ist es, Wasser auf $-5^{\circ}$ bis $-10^{\circ}$ zu unterkühlen; wer je mit BunsEN's Eiskalorimeter gearbeitet hat, wird diese Erfahrung gemacht haben, wenn er den Apparat nur mit möglichst sorgfältig gereinigtem Wasser beschickt hatte.

Andere Beispiele, die uns gerade einfallen, sind das Monokaliumphosphat und das Monokaliumarseniat. Diese Salze, besonders das letztere, bilden aufserordentlich leicht übersättigte Lösungen, eine heilsgesättigte Lösung kann man auf $0^{\circ}$ abkühlen, ohne dals Ausscheidung erfolgt; erst bei heftigem Schütteln tritt Krystallisation ein. Von einer zweiten Modifikation oder von einem Hydrat ist weder beim $\mathrm{KH}_{2} \mathrm{PO}_{4}$ noch beim $\mathrm{KH}_{2} \mathrm{AsO}_{4}$ etwas bekannt.

Wahrscheinlich würde Herr Nicou niemals auf den Gedanken gekommen sein, obigen Satz zu behaupten, wenn er eine richtige Definition für das Wort „Übersättigung“ aufgestellt hätte. Er spricht in der ganzen Abhandlung von übersättigten Lösungen schlechthin und erwähnt mit keinem Wort, dafs eine Lösung nur in Bezug auf eine $\mathrm{Phase}$ nicht aber in Bezug auf einen der Stoffe übersättigt sein kann. An einem instruktiven Beispiele möge klar gemacht werden, was wir sagen wollen:

Man löse in $100 \mathrm{~g}$ Eiswasser $8 \mathrm{~g}$ wasserfreies $\mathrm{Ce}_{2}\left(\mathrm{SO}_{4}\right)_{3}$ auf, bringe das Kölbchen verschlossen in einen Thermostaten und erwärme langsam, unter Vermeidung von Erschütterung, auf 50 . Dann werfe man vorsichtig einige frisch bereitete Krystalle des Oktohydrats in die Lösung und erhalte die Temperatur eine Zeit lang konstant. Die Krystalle verschwinden; nach kurzer Zeit sind sie völlig aufgelöst.

Nun stelle man denselben Versuch an mit dem Pentahydrat. Die Nadeln fallen zunächst zu Boden; nach kurzer Zeit bemerkt man aber, wie in der Flüssigkeit Kryställchen entstehen, die nach 
einiger Zeit sich absetzen. Die Versuche gelingen nur, wenn die zum „Impfen" benutzten Krystalle ganz frisch bereitet waren.

Was ist nun die Lösung, die solche Eigenschaften zeigt; ungesättigt oder übersättigt? Doch offenbar beides zu gleicher Zeit, es kommt blofs auf die feste Phase an, die man mit ihr in Berührung bringt.

Welche Wirkung die blofse Existenzfähigkeit des Oktohydrats haben soll auf die Eigenschaft der Lösung, Krystalle des Pentahydrats beim Impfen mit diesem Salze auszuscheiden, vermögen wir absolut nicht einzusehen. Das Oktohydrat hat mit der ganzen Erscheinung nicht das Mindeste zu thun; und ähnlich ist es bei den Beispielen, welche Herr Nrcor in seiner Abhandlung beschreibt.

München, Chem. Laboratorium der kgl. Akademie der Wissenschaften.

Bei der Redaktion eingegangen am 25. Januar 1898. 